\title{
Excitation power dynamics of photoluminescence in InGaN/GaN quantum wells with enhanced carrier localization
}

\author{
K. Kazlauskas, a) G. Tamulaitis, J. Mickevičius, E. Kuokštis, and A. Žukauskas \\ Institute of Materials Science and Applied Research, Vilnius University, Sauletekio 9-III, LT-10222 Vilnius, \\ Lithuania \\ Yung-Chen Cheng, Hsiang-Cheng Wang, Chi-Feng Huang, and C. C. Yang \\ Graduate Institute of Electro-Optical Engineering and Department of Electrical Engineering, National \\ Taiwan University 1, Roosevelt Road, Section 4, Taipei, Taiwan, Republic of China
}

(Received 9 June 2004; accepted 4 October 2004; published online 14 December 2004)

\begin{abstract}
Excitation-power dynamics of near-band-edge photoluminescence (PL) peak position in $\mathrm{In}_{x} \mathrm{Ga}_{1-x} \mathrm{~N} / \mathrm{GaN}$ multiple quantum wells $(x \sim 0.15)$ was analyzed as a function of well width. The analysis was based on energy reference provided by photoreflectance (PR) spectra. The difference in spectral position of the PR feature and low-excitation PL band (the Stokes Shift) revealed carrier localization energy, which exhibited a remarkable sensitivity to the well width, increasing from $75 \mathrm{meV}$ in $2 \mathrm{~nm}$ wells to about $250 \mathrm{meV}$ in $4 \mathrm{~nm}$ wells. Meanwhile collating of the PR data with the flat-band model for the optical transition energy in quantum wells rendered a relatively weak $(0.5 \mathrm{MV} / \mathrm{cm})$ built-in piezoelectric field. The blueshift of the PL peak position with increasing photoexcitation power density was shown to be in qualitative agreement with the model of filling of the band-tail states with some contribution from screening of built-in field in the thickest $(4 \mathrm{~nm})$ wells. Increased incident photon energy resulted in an additional blueshift of the PL peak, which was explained by a nonthermalized distribution of localized carriers and/or carrier localization in the interface region. Our results are consistent with a concept of emission from partially relaxed large In-rich regions with internal band potential fluctuations, which are enhanced with increasing the growth time. () 2005 American Institute of Physics. [DOI: 10.1063/1.1826220]
\end{abstract}

\section{INTRODUCTION}

InGaN compounds are already acknowledged as key materials in many commercial green-blue light-emitting devices such as light-emitting diodes and laser diodes. ${ }^{1-3}$ Many groups have extensively studied the InGaN-related epilayers and structures containing quantum wells from the viewpoint of the specific nature of optical transitions at the fundamental absorption edge. ${ }^{4-20}$ However, a few essential problems still remain unresolved, in particular, those regarding the emission spectral and temporal dynamics with temperature, nonequilibrium carrier density, quantum-well width, and In molar fraction. A number of investigators came to a conclusion that in indium containing nitride compounds, indium atoms are nonhomogeneously distributed with pronounced Incontent fluctuations. ${ }^{4-6,11,13,20}$ This inhomogeneity results in localization of nonequilibrium carriers and, as generally believed, enhances performance of InGaN-based light-emitting devices. ${ }^{11}$ However in multiple quantum wells (MQWs), which are commonly used in the active region of InGaNbased light-emitting devices, the efficiency of light emission is believed to be considerably inhibited by quantum-well potential tilting, which results in electron and hole wave function separation (quantum confined Stark effect, QCSE) ${ }^{8,21}$ In InGaN, the QCSE is caused by built-in electric field mainly due to strain-induced piezoelectric polarization at wellbarrier interfaces. ${ }^{22}$

${ }^{a)}$ Electronic mail: karolis.kazlauskas@ff.vu.lt
Correspondingly, present understanding of the origin of near-band-edge optical transitions in InGaN quantum wells relies on several models. ${ }^{11,23}$ First model employs exponential band tails formed by compositional fluctuations of In content with carriers or excitons residing within energy levels confined in the fluctuation minima. ${ }^{4,6,11}$ An extension of this model is complete confinement of carriers in the small $\left(\sim 1 \mathrm{~nm}\right.$ size) In-rich clusters ( $\mathrm{InN}$ quantum dots) ${ }^{5,13}$ which are formed by phase separation peculiar to the InGaN system. $^{24,25}$ The quantum-dot localization model receives somewhat less experimental support and contradicts to some experimental data. In particular, our recent results on Monte Carlo simulation of exciton/carrier hopping in In-containing nitride alloys revealed clear spectroscopic signatures of the exciton in-plane motion over an ensemble of localized states within isolated regions, which, in turn, exhibit some fluctuation in the net band gap energy (double-scaled band potential profile) ${ }^{26,27}$ This provides support for the model of extended $(<10 \mathrm{~nm})$ In-rich regions with a rough internal band potential profile ("quantum discs" or "segmented quantum wells") as reviewed in Ref. 11.

In the alternative model, strong built-in field is assumed to govern dynamics of the emission mechanism via the QSCE. Rather high magnitudes of the built-in electric field strength (about $1.5-2 \mathrm{MV} / \mathrm{cm}$ ) are expected for InGaN/GaN quantum wells containing $\sim 15 \%$ indium. ${ }^{22,28}$ Even higher values of $2.5-3 \mathrm{MV} / \mathrm{cm}$ were extracted from the variation of the photoluminescence (PL) peak position with the well width, assuming that the localization energy is 
well-width independent. ${ }^{14,29,30}$ However, both localization at the band-tail states and the QCSE result in similar dynamics of the luminescence spectra and are difficult to distinguish. In particular, a blueshift of the PL line with increased carrier density as well as an increase of the luminescence decay time with reduced optical transition energy can be caused by either screening of the built-in field ${ }^{31}$ or band-tail filling. ${ }^{7}$ Again, a widely accepted belief in that, redshifting of the emission line and increasing of the decay time with the well width are the signatures of solely the QCSE, is based on an assumption that increased well width results in no structural changes of the InGaN material. Meanwhile, there exist clear indications that increased growth time may result in dramatic redistribution of indium in the wells due to thermally invoked In-Ga interdiffusion, ${ }^{20,32}$ and even in graded average In content. ${ }^{33}$ Even more intricate behavior of emission from InGaN was observed by using PL spectroscopy with subwavelength resolution, which revealed energy shifting of single localized states with temperature and no shift with excitation power. $^{34}$

To provide with an improved physical background for further optimization of InGaN-based light emitters, it is therefore important to work out experimental methods for distinguishing between the localization effects and built-in field effects. Sophisticated experiments involving quaternary barriers, ${ }^{35}$ scaling to strain-free epilayers ${ }^{18}$ and time-resolved cathodoluminescence $^{36}$ have shown that both the QCSE and localization effect might modify the optical properties of Incontaining nitride structures to a comparable extent.

In this paper, our analysis of well-width dynamics of the PL spectra in InGaN/GaN MQWs is based on spectroscopic reference provided by photoreflectance (PR) data. A similar modulation-spectroscopy (electroabsorption) approach employing distinct resonance features was initially introduced for proving of carrier localization effects in InGaN. ${ }^{11}$ However, such resonance spectroscopy is being usually forsaken and substituted by simplified approaches such as identifying of the PL peak position with the fundamental optical transition energy ${ }^{29,30}$ or extracting of the "effective band gap" from broad tails of the absorption spectra. ${ }^{13}$ PR features due to quantum wells are known to strictly follow the QCSE induced reduction of the optical transition energy, as it has been revealed in InGaAs/GaAs structures. ${ }^{37}$ Here, based on the PR reference in InGaN/GaN MQWs with different well width, we directly distinguished between the PL redshift caused by localization of nonequilibrium carriers due to band potential fluctuations and well potential tilting due to built-in electric field. This enabled us to perform a reliable analysis of excitation-power dynamics of the luminescence peak, which in the particular set of samples was shown to be mainly governed by filling of band-tail states. In addition, the band-tail filling effect was found to be sensitive to the excitation photon energy.

\section{EXPERIMENT}

We have investigated $\operatorname{In}_{x} \mathrm{Ga}_{1-x} \mathrm{~N} / \mathrm{GaN}$ MQWs, which were grown by metalorganic chemical vapor deposition (MOCVD) over (0001) surface of sapphire substrate covered by $30 \mathrm{~nm}$ low-temperature layer and a $2.3 \mu \mathrm{m}$ thick buffer layer of $n-\mathrm{GaN}$. The ratio of the In and $\mathrm{Ga}$ precursor flows was set for In molar fraction $x$ of 0.15 . The samples consisted of five InGaN/GaN quantum wells separated by $10 \mathrm{~nm}$ thick GaN barriers. A set of five samples with the different well widths $L_{z}$ of 2.0, 2.5, 3.0, 3.5, and $4.0 \mathrm{~nm}$ were grown in otherwise identical conditions.

The PL and PR spectra were measured using a Jobin Yvon double monochromator HRD-1 and a photomultiplier. In the PR experiments, chopped radiation of the $\mathrm{He}-\mathrm{Cd}$ laser emitting at $325 \mathrm{~nm}(3.815 \mathrm{eV})$ was used for excitation and a halogen lamp was used as a source of reflected light. The modulated component of the reflectance signal was extracted using a lock-in amplifier. For some samples, additional modulation of the reflected light was applied to remove the background PL. PL spectra under continuous wave (cw) excitation were recorded in the photon counting mode.

A pulsed organic-dye laser pumped by the third harmonic of YAG: $\mathrm{Nd}^{3+}$ (YAG-yttrium aluminum garnet) laser radiation was applied in order to study $\mathrm{PL}$ dynamics under moderate to high excitation and different photon energies. The pulse duration of $10 \mathrm{~ns}$ ensured quasi-steady-state excitation conditions. Two excitation photon energies were employed. Photons with the energy $h \nu_{\mathrm{exc}}=3.255 \mathrm{eV}$, which is below the band gap of $\mathrm{GaN}$, excited carriers only in the InGaN quantum wells (selective excitation), whereas both the wells and barriers were excited by photons with $h \nu_{\text {exc }}$ $=3.625 \mathrm{eV}$ (unselective excitation). In the pulsed mode, the spectra were taken using a boxcar integrator.

\section{EXPERIMENTAL RESULTS}

The PR and PL spectra of the samples were dominated by the near-band-gap optical transitions and we will concentrate our attention exactly upon this spectral region. (Besides this emission, we observed a broad yellow deep-level luminescence band; its intensity is higher in samples with larger well widths.) The normalized PR and low-excitation PL spectra of InGaN/GaN MQWs with different well width are depicted in Fig. 1. Arrows in Fig. 1 denote the energy of the average band gap $E_{0}$ deduced from the PR spectra ${ }^{38,39}$ within a conventional Lorentzian functional approach. Note that this energy is inherently affected by the QCSE, i.e., it is already reduced in comparison with that for the flat-band model. ${ }^{37}$ With decreasing the well width, both the PR feature and PL band peak position shift towards short wavelengths, probably due to quantum confinement effect. The specific feature of these data is a pronounced redshift of the PL peak with respect to the fundamental optical transition. To avoid confusion with diverse description of the PL spectral behavior in polarized quantum wells, here we denominate this shift as a spectroscopically confident Stokes shift $\Delta E_{S}$, which indicates that emission originates from localized states. ${ }^{11}$

Figure 2(a) depicts the well-width dependence of the PR (filled points) and low-excitation PL (open points) data. Note that the broad PR features observed in our spectra can be not only due to fundamental optical transition $11 \mathrm{H}$ but also contain an admixture of the symmetry-forbidden optical transition $12 \mathrm{H}$ involving first excited hole level, which has a dif- 


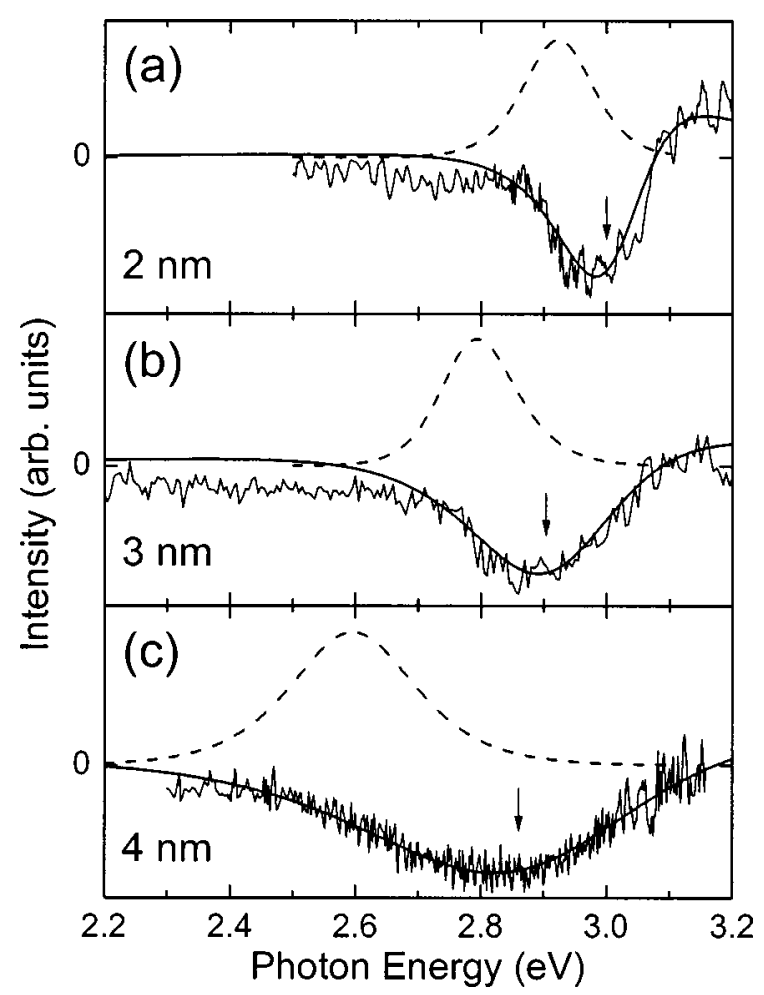

FIG. 1. PR (solid lines) and PL (dashed lines) spectra of InGaN/GaN MQW structures with the well width of 2.0 (a), 3.0 (b), and $4.0 \mathrm{~nm}$ (c). The PR spectra are fitted with Lorentzian line shape functional form. Arrows indicate the energy of the fundamental optical transition.

ferent sensitivity to band tilting. ${ }^{37}$ For reasonable values of the built-in field $(<1 \mathrm{MV} / \mathrm{cm})$, the resulting uncertainty of the average band gap is reflected by asymmetric error bars in Fig. 2(a). Since this uncertainty is smaller than the width of the PR feature and much smaller than the observed Stokes shift, below we neglect the effects due to the $12 \mathrm{H}$ transition.

The confident Stokes shift in Fig. 2(a) is seen to monotonously increase from $75 \mathrm{meV}$ to about $250 \mathrm{meV}$ when the well width increases from $2.0 \mathrm{~nm}$ to $4.0 \mathrm{~nm}$. Enhancement of the Stokes shift with increasing the well width might be attributed to an enhancement of the band potential fluctuations, which the carriers are localized at. The assumption on increased localization depth is supported by that an increased well width results in an increased spectral width of the PR feature due to a larger dispersion of the band-tail states within the In-rich regions as shown in Fig. 1. Broadening of the PL band from about $130 \mathrm{meV}$ in $2 \mathrm{~nm}$ wells to $230 \mathrm{meV}$ in $4 \mathrm{~nm}$ wells is also clearly seen. Note that we did not observe a significant decrease in PL intensity with increasing the well width, as might be expected due solely to spatial separation of electrons and holes caused by the QCSE. This is one of the indications that the emission efficiency in our samples is governed by carrier localization rather than by electron-hole wave function separation.

Figure 3 shows the PL spectra families recorded under different pulsed excitation power density for two samples with the well width of $2.0 \mathrm{~nm}$ and $4.0 \mathrm{~nm}$. The spectra presented in Figs. 3(a) and 3(c) were taken under unselective excitation with photons absorbed both in the well and barrier layers $\left(h \nu_{\mathrm{exc}}=3.625 \mathrm{eV}\right)$, whereas the spectra in Figs. 3(b)

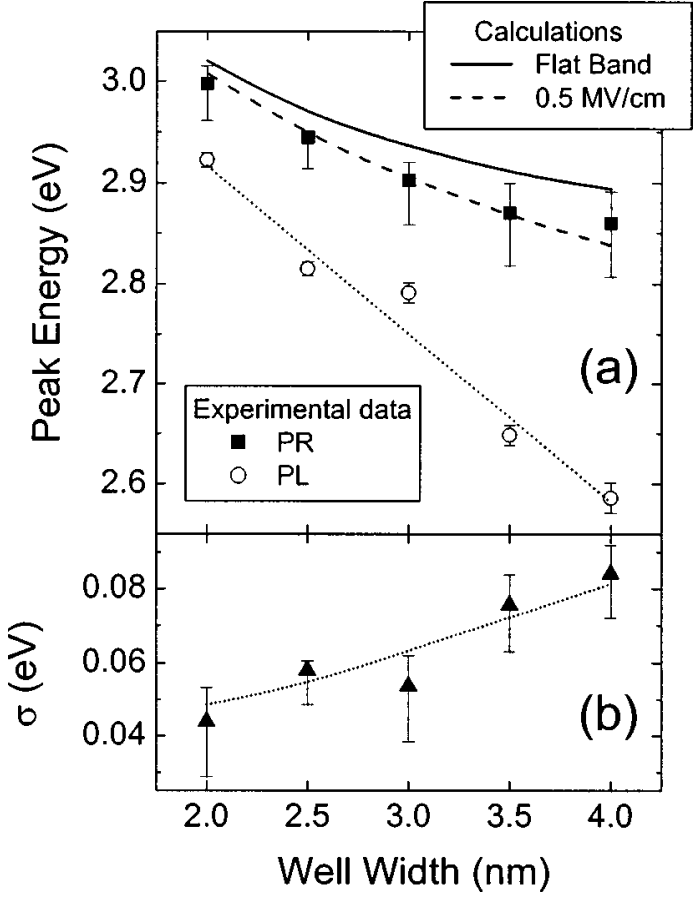

FIG. 2. (a) Transition energy deduced from the PR spectra (filled points) and PL peak position (open points with a dotted guide for the eye) as a function of well width in InGaN/GaN MQWs. Dashed line, the calculated energy of the fundamental optical transition for the tilted wells with the built-in field strength of $0.5 \mathrm{MV} / \mathrm{cm}$ and the indium molar fraction of $x$ $=0.165$; solid line, same for rectangular wells. (b) Band potential profile roughness as a function of well width deduced from the difference in energy of the PR and PL data.

and 3(d) and were measured under selective excitation of solely the wells with photons of the energy $h \nu_{\mathrm{exc}}=3.255 \mathrm{eV}$. In the former case, the two separate PL bands can be clearly resolved [Figs. 3(a) and 3(c)]. When selective excitation of the wells is applied, the PL spectrum is dominated by a single band. Obviously, this PL band, as well as the longwavelength band under unselective excitation is caused by radiative recombination in InGaN wells. Meanwhile, the short-wavelength band peaked at $\sim 3.42 \mathrm{eV}$ is due to the photoexcited carriers generated in the $\mathrm{GaN}$ barrier/buffer layers and recombining before their relaxation into the well.

As one can see in Fig. 3, an increased excitation power density results in a blueshift of the peak position and broadening of the InGaN-well related band, meanwhile the position and shape of the GaN barrier/buffer band remains almost unchanged. Data extracted from Fig. 3 are presented in more detail in Fig. 4 with the different photogeneration efficiencies for two incident photon energies taken into account. Here, points reproducing data for different excitation photon energies (open points for nonselective excitation and filled points for selective excitation) are arbitrarily shifted to achieve coincidence in the PL intensity and, consequently in carrier density, assuming that the same PL intensity approximately corresponds to the same carrier concentration for both the excitation photon energies. In our case, matching the PL intensities means that photoexcitation of both the InGaN well and the $\mathrm{GaN}$ barrier/buffer layers results in about 4-fold increase in carrier net generation rate in the wells as compared to selective photoexcitation of the $\mathrm{InGaN}$ well layers. 


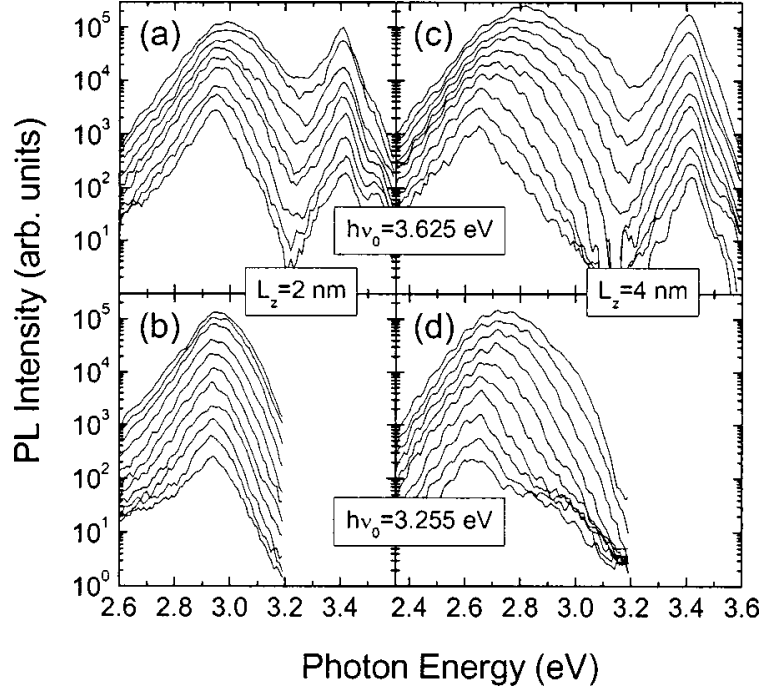

FIG. 3. PL spectra of InGaN/GaN MQWs with the well width of $2.0 \mathrm{~nm}$ (a), (b) and $4.0 \mathrm{~nm}$ (c), (d) at the excitation photon energy of $3.625 \mathrm{eV}$ (a), (c) and $3.255 \mathrm{eV}$ (b), (d) measured as a function of excitation power density. The excitation power density is incremented by a factor of 2 up to $2 \mathrm{MW} / \mathrm{cm}^{2}$

Figures 4(a) and 4(b) show that the PL intensity dependence on the excitation power density in different samples under selective, as well as unselective, excitation is close to linear within the range of three orders of magnitude up to the highest excitation densities used $\left(2 \mathrm{MW} / \mathrm{cm}^{2}\right)$. This suggests that the dominant emission mechanism is bimolecular recombination. ${ }^{40}$ Indeed, since quantum structures are rather thin (the total thickness of the MQW "sandwich" is usually less than a typical absorption depth of the excitation light $\alpha^{-1}$ ), we can assume homogeneous excitation of the layers. Thus for bimolecular recombination, the photogenerated carrier density increases as $n=\sqrt{\alpha P / h \nu_{\text {exc }} B}$, where $P$ is the excitation power density (in $\mathrm{W} / \mathrm{cm}^{2}$ ) and $B$ is the recombination coefficient $\left(\right.$ in $\left.\mathrm{cm}^{3} / \mathrm{s}\right){ }^{40}$

Excitation-photon dependent dynamics of the InGaNrelated PL peak position for two different samples shown in Figs. 4(c) and 4(d) illustrates that at low excitation the peak position of the PL band is independent of the excitation photon energy. However, with increasing the excitation power under almost identical carrier generation rates, the blueshift is larger when excitation with the higher photon energy is applied. Another essential result is that blueshifting is more pronounced in samples with wider wells. Similar behavior is peculiar to dynamics of the PL linewidth with the excitation power density (not shown). The linewidth increases with the excitation power density and this increase is more noticeable in wider wells and for the larger incident photon energy.

\section{MODELS AND CALCULATIONS}

The experimentally observed excitation power-induced shift of the PL band towards short wavelengths in MQWs might be caused by (i) filling of the band-tail localized states and (ii) screening of the built-in electric field by the photoexcited carriers, which results in the reduced QCSE. Both effects necessitate the same shift sign and it is challenging to distinguish them experimentally. ${ }^{18}$ To get a deeper insight

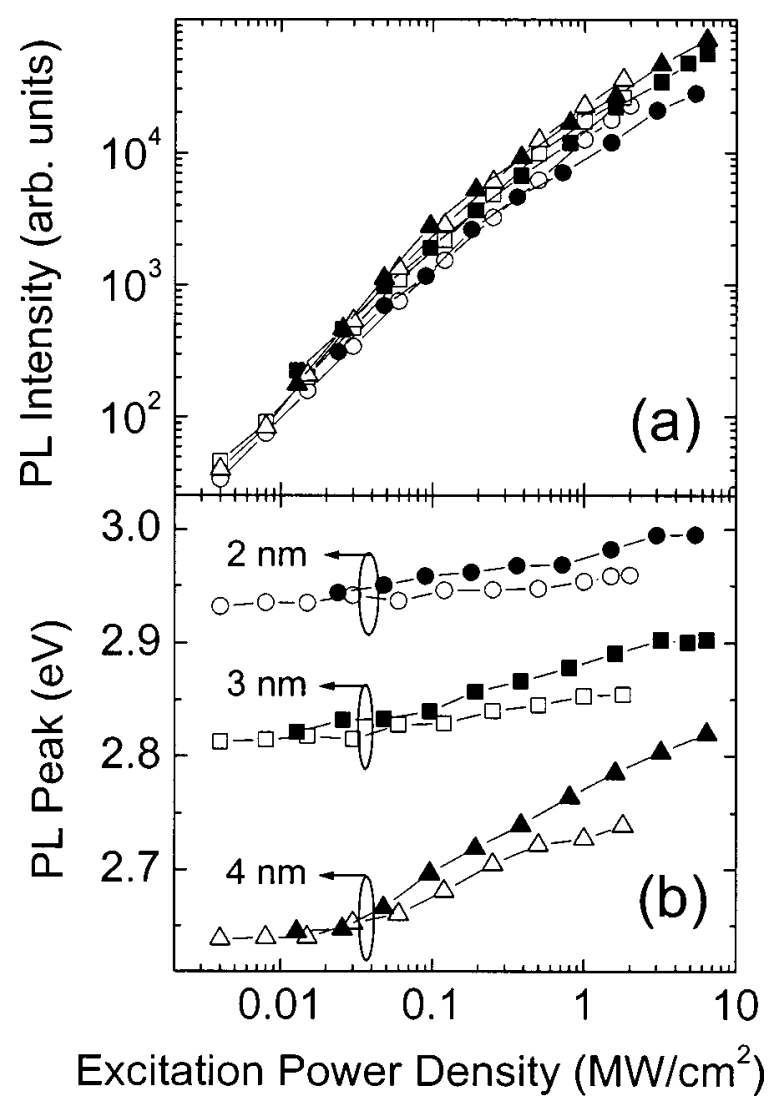

FIG. 4. Excitation power density dependence of spectrally integrated PL intensity (a) and PL band peak position (b) under excitation at $3.255 \mathrm{eV}$ (open points) and $3.625 \mathrm{eV}$ (filled points) in InGaN/GaN MQWs. The dependences for the different incident photon energies are shifted along the horizontal axis to achieve coincidence in PL intensity. Squares, triangles, and circles correspond to the well width of $2.0 \mathrm{~nm}, 3.0 \mathrm{~nm}$, and $4.0 \mathrm{~nm}$, respectively.

into the band-tail filling and built-in field screening effects, we performed theoretical simulation of the PL peak dynamics with the excitation power density for the two models. Points in Fig. 5 show the experimental dependence over the excitation power density range of 9 orders of magnitude with both the low-excitation (cw) and high-excitation (pulsed unselective) data included. To perform qualitative calculations, two quantities are needed to be estimated, namely, the roughness of the band potential profile $\sigma$ and the strength of the initial built-in field $F$, respectively.

The roughness of the band potential profile, which relates to the effective dispersion $\sigma^{2}$ of the Gaussian tails for electrons $\left(\sigma_{e}^{2}\right)$ and holes $\left(\sigma_{h}^{2}\right)$ as $\sigma^{2}=\sigma_{e}^{2}+\sigma_{h}^{2}$, can be easily estimated under assumption of thermalized distribution of carriers over the band-tail states. Under such conditions, the effective dispersion is related to the low-excitation Stokes shift as ${ }^{7,27}$

$$
\Delta E_{S}=\sigma^{2} / k_{B} T .
$$

Figure 2(b) shows the roughness as a function of well width. The roughness is seen to monotonously increase from $44 \mathrm{meV}$ in the $2 \mathrm{~nm}$ wells to $84 \mathrm{meV}$ in 4 nm wells. Such an increase in dispersion of the band-tail states with the well width is probably caused by enhanced segregation of indium with increased time of growth, ${ }^{32}$ what results in an enhancement of band potential fluctuations. 


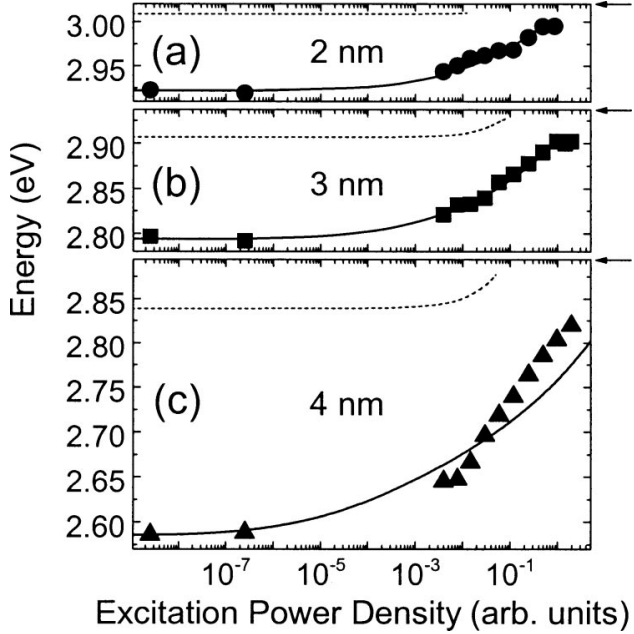

FIG. 5. PL peak position vs excitation power density for InGaN/GaN MQWs with the well width of 2.0 (a), 3.0 (b), and $4.0 \mathrm{~nm}$ (c). Solid lines, calculation for the band-tail filling effect; dashed lines, the effect of screening of built-in field. Arrows indicate the energy of the lowest optical transition in the rectangular wells.

The blueshift due to band-tail filling as a function of carrier density was calculated from the spontaneous emission spectrum with no momentum conservation ${ }^{7}$

$$
r_{s p}(h \nu)=B \int_{-\infty}^{\infty} \rho_{e}(E+h \nu) f_{e}(E+h \nu) \rho_{h}(E) f_{h}(E) d E,
$$

where the recombination coefficient $B$ is assumed to be independent of the photon energy. The densities of the bandtail states for electrons and holes, $\rho_{e}(E)$ and $\rho_{h}(E)$, respectively, are described by the Gaussian functions

$$
\rho_{e, h}(E)=\frac{N_{0}}{(2 \pi)^{1 / 2} \sigma_{e, h}} \exp \left[-\left(E-E_{0 e, h}\right)^{2} / 2 \sigma_{e, h}^{2}\right],
$$

where $N_{0}$ is the total number of the localized states and $E_{0 e}$ and $E_{0 h}$ are the central energies for the conduction and valence band, respectively $\left(E_{0 e}-E_{0 h}=E_{0}\right)$. To account for the band filling, we used Fermi-Dirac distribution functions $f_{e, h}(E)=\left\{\exp \left[\left(E-F_{e, h}\right) / k_{B} T\right]+1\right\}^{-1}$, where $F_{e}$ and $F_{h}$ are the Fermi quasilevels for electrons and holes, respectively.

For a particular carrier density $n$, the Fermi quasilevels were extracted using a relation $n=\int_{0}^{\infty} \rho_{e, h}(E) f_{e, h}(E) d E$ and the emission spectrum was calculated using Eq. (2). In the calculations, we used a ratio of $\sigma_{h} / \sigma_{e}=0.1$ in accordance with the valence to conduction band offset ratio. The resulting dependences of the emission band peak vs excitation power density for the three samples are presented in Fig. 5 by solid lines. The dependences were fitted to the experimental points by shifting along the horizontal axis through adjusting of the total number of the localized states $N_{0}$. This number was almost independent of the well width and equaled to about $7 \times 10^{19} \mathrm{~cm}^{-3}$.

To reveal the value of built-in field, the experimentally obtained dependence of the fundamental optical transition energy on the well width [Fig. 2(a)] was compared with calculations for two models involving rectangular (flat-band) and triangular (tilted-band) quantum wells, respectively. The calculations were based on solution of the Schrödinger equa- tion and accomplished by using the Numerov technique. ${ }^{41}$ Solid line in Fig. 2(a) shows the result of calculation for rectangular wells. In calculations, we have used PR data on the band gap energy of InGaN coherent with the GaN buffer, ${ }^{38} 0.535 \mathrm{eV}$ and $0.045 \mathrm{eV}$ band offsets for the conduction and valence band, respectively, ${ }^{42}$ and values of $0.16 m_{0}$ and $1.73 m_{0}$ for the electron and hole masses, respectively. ${ }^{43,44}$ The calculated tilted-band dependence was fitted to the experimental points of the fundamental optical transition energy by adjusting two parameters, the In molar fraction $x$ and the field strength $F$. A satisfactory fit with the PR data was achieved for $x=0.165$ and $F=0.5 \mathrm{MV} / \mathrm{cm}$.

To account for screening of built-in electric field by the photoexcited carriers, the Schrödinger and Poisson equations were solved self-consistently. ${ }^{45,46}$ Solution of the Schrödinger equation with the initial well-bottom potential energy $V_{0}(z)$, which is determined by unscreened built-in field, yielded the wave functions $\Psi_{e, h}^{(i)}(z)$ in the energy level $i$. With band potential fluctuations across the well neglected, the resulting spatial distribution of electrons and holes in the well is

$$
n_{e, h}(z)=\sum_{i} n_{e, h}^{(i)}\left|\Psi_{e, h}^{(i)}(z)\right|^{2},
$$

where $n_{e, h}^{(i)}$ is the electron (hole) density in the energy level $i$, which depends on the total density of the photogenerated carriers. The spatial distribution of electrons and holes alters the well-bottom potential profile by a variation $\phi(z)$, which can be calculated from the Poisson equation

$$
\frac{d}{d z}\left[\varepsilon(z) \frac{d \phi(z)}{d z}\right]=\frac{e\left[n_{e}(z)-n_{h}(z)\right]}{\varepsilon_{0}},
$$

where $\varepsilon(z)$ is the dielectric permittivity (considered to have constant values in the barrier and well layers). Equation (5) was segmented and solved by using the tridiagonal matrix technique. The modified potential energy profile $V^{\prime}(z)$ $=V_{0}(z)-e \phi(z)$ was used to iteratively solve the Schrödinger and Poisson equations until the variation $\phi(z)$ changed negligibly.

Dashed lines in Fig. 5 show the calculated variation of the electron-hole transition energy for the lowermost subbands $(i=1)$ due to screening of built-in field with increasing the excitation power density. Again, here we assumed the domination of bimolecular recombination of the nonequilibrium carriers, i.e., the carrier density increases as a square root of the excitation power density. The difference in transition energy between the low-excitation and high-excitation limits equals the difference in energy between the flat-band and tilted-band models as revealed in Fig. 2(a).

\section{DISCUSSION}

The considerations presented above have shown that analysis of PL peak dynamics in InGaN/GaN MQWs cannot be based solely on the PL spectroscopic data. Actually, the results on the PL band broadening and redshifting with the well width as well as on its blueshifting with the excitation power density can be accounted for not only by the band-tail filling effect, but also in terms of the QCSE with screening of 
built-in field assuming higher values of the initial field strength (2.5-3 MV/cm). Stronger field should cause a larger redshift, and larger fluctuations of the field should result in a broader PL band. Also, the excitation power dependence of the PL peak blueshifting due to screening (see Fig. 5 ) is qualitatively similar to that due to the band-tail filling effect and may occur in the same range of carrier densities.

However, spectroscopic reference provided by the PR data delineates a ridge between the two effects. By using this reference, one can clearly extract the confident Stokes shift, which is related solely to the effect of carrier localization in the band potential fluctuation minima. Simultaneously, the difference in energy between the rectangular-well band gap and the PR feature yields the shift due to the QCSE. Although some uncertainty in determination of indium molar fraction and calibration of the bandgap energy in InGaN may exist, this issue can be resolved using a well-width dependence of the PR spectrum as was shown above.

Large dispersion of localized-state energy in our samples governs not only the peak position of the PL band, but also its full width at half maximum (FWHM) at low excitation. Under assumption of thermalized distribution of carriers over the band-tail states in the absence of band-tail filling effect, the homogeneous FWHM is ${ }^{7,27} \gamma=2 \sqrt{\ln 4} \sigma$ with some contribution of phonon broadening of $\leqslant k_{B} T$ in magnitude. For instance, in the thinnest $(2 \mathrm{~nm})$ and thickest $(4 \mathrm{~nm})$ wells studied in this work, the value of $\sigma[44 \mathrm{meV}$ and $84 \mathrm{meV}$, Fig. 2(b)], should yield the PL band FWHM of about $100 \mathrm{meV}$ and $200 \mathrm{meV}$, respectively. However, in indium containing group-III nitride alloys, the PL band is usually inhomogeneously broadened. In thick films, inhomogeneous broadening $\Gamma_{0}$ can be attributed to dispersion in the net band gap energy of individual In-rich regions due to macroscopic fluctuations in In content. ${ }^{27}$ In quantum wells, additional factors of PL band broadening might be important. In particular, fluctuations in well width result in a variation of quantum confinement energy $\Gamma_{q}$. For the well-width variation $\delta L_{z}$ within one monolayer $(0.265 \mathrm{~nm})$, our estimations yield the values of $\Gamma_{q}$ of about $30 \mathrm{meV}$ and $10 \mathrm{meV}$ for $2 \mathrm{~nm}$ and $4 \mathrm{~nm}$ wells, respectively. Probably, this effect can noticeably manifest itself for the well width below $2 \mathrm{~nm}$. Additionally, the redshift due to the QCSE is modulated by lateral fluctuations in indium content, which results in a variation of piezoelectric field, and by the variation of the well width. The resulting inhomogeneous broadening, ${ }^{47} \quad \Gamma_{F}=e \sigma L_{z} \partial F / \partial E_{g}$ $+e F \delta L_{z}$, yields for our samples the values of $22 \mathrm{meV}$ and $47 \mathrm{meV}$, respectively, for $F=0.5 \mathrm{MV} / \mathrm{cm}$ and $\partial F / \partial E_{g}$ $\approx 1 \mathrm{MV} / \mathrm{cm}$ eV. Meanwhile, the overall PL band FWHM is

$$
\mathrm{FWHM} \approx\left[\gamma^{2}+\left(k_{B} T\right)^{2}+\Gamma_{q}^{2}+\Gamma_{F}^{2}+\Gamma_{0}^{2}\right]^{1 / 2} .
$$

For the actual FWHM of the PL band of $130 \mathrm{meV}$ and $230 \mathrm{meV}$ for the $2 \mathrm{~nm}$ and $4 \mathrm{~nm}$ wells, Eq. (6) yields the values for $\Gamma_{0}$ of about $70 \mathrm{meV}$ and $100 \mathrm{meV}$, respectively. The above considerations indicate that in our samples, the PL linewidth is due mainly to potential roughness within the In-rich regions and dispersion in the net band gap energy of individual In-rich regions. Furthermore, increased dispersion of the band-tail states in thicker wells is probably also the main reason for broadening of the PR feature.
Based on the spectroscopic partition of the initial redshift of the PL band between the band-tail filling effect and the QCSE, dynamics of the excitation power dependence of the PL peak position can be understood. The results of calculation presented by lines in Fig. 5 infer that in our samples, blueshifting with the excitation power density is dominated by band-tail filling. Effect of flattening of the well bottom might contribute noticeably only in the thickest $4 \mathrm{~nm}$ wells, where the calculated blueshifting due to band-tail filling is insufficient to account for the experimental dynamics. It should be noted that the described dynamics of the PL peak position is peculiar to certain samples such as those used in our work. These MOCVD-grown samples feature broad PL lines, a pronounced dependence of the PL linewidth and redshift on the well width, and well-width dependent optical properties even at extremely high photoexcitation when the built-in field is expected to be completely screened. ${ }^{48}$ Our analysis of extended literature data implies that the domination of the band-tail filling effect over screening of built-in field might be not a universal property of luminescence dynamics in InGaN quantum wells. The situation might be quite converse in samples, which exhibit narrow PL lines indicating smaller fluctuations of In molar fraction and smoother band potential profile. Note that our data is in line with a relatively low strength of built-in field $(0.5 \mathrm{MV} / \mathrm{cm})$, which is consistent with some other experimental data for MOCVD-grown structures ${ }^{10}$ but somewhat disagrees with theoretical expectations $(\sim 2 \mathrm{MV} / \mathrm{cm}) .{ }^{22,28}$ However, the latter expectations rely on an assumption of uniformity of the InGaN alloy, whereas within the In-rich regions, where the emission probably comes from, the field strength can be different. We attribute the reduced value of the built-in field strength to partial strain relaxation within the In-rich regions.

Behavior of the band-tail filling effect with variation of the excitation photon energy requires special consideration. Data presented in Fig. 4 show that both the photon energies used result in the same PL peak position for low excitation, what indicates that in both cases excitation above the mobility edge was performed. ${ }^{49}$ An increase in the excitation power density under the same carrier generation rate results in faster blueshifting for the larger incident photon energy. This can be an indication of the built-in field screening effect, since larger excitation photon energy facilitates generation of delocalized carriers, which contribute to screening more effectively than localized ones owing to free motion. However, based on the above considerations, such an explanation should be ruled out for our samples because of the negligible contribution of the QCSE to the initial redshift of the PL band. As an alternative, we suggest that increased incident photon energy modifies the band-tail filling effect because of two possible reasons. First, a nonthermalized distribution of nonequilibrium carriers can occur due to a large excess energy released during the energy relaxation of the photoexcited carriers. The larger excess energy results in nonequilibrim ("hot") phonon population, ${ }^{50}$ which can shift the carrier distribution over the localized states to higher energies. Second, when barriers are involved in the photoexcitation, the spatial distribution of the nonrelaxed carriers is different from that in the case of selective excitation of the 
wells and the carriers can undergo different routes of localization in In-rich regions. As demonstrated by highresolution transmission electron microscopy, the In-rich regions tend to form at the well-barrier interface and extend into the barrier, especially when the average In content is increased above $15 \% .{ }^{20}$ Such an extension of the In-rich regions into the barriers facilitates localization of the carriers, which are excited in the barriers, directly at the interfaces. Localized states at the interfaces can be also produced by formation of cubic-GaN regions. ${ }^{51}$ As a result, a wider nomenclature of the tail states with a shift to higher energies might be involved in the localization process in comparison with that for the case of selective excitation solely in the wells.

In summary, spectroscopic reference provided by the PR spectra enabled us to distinguish between the carrier localization effect and the QCSE in InGaN/GaN MQWs of different well width $(2-4 \mathrm{~nm})$. Specifically to our MOCVDgrown samples with a broad spectrum of localized states, an increase in localization energy with increasing the well width was revealed and the strength of built-in field $(0.5 \mathrm{MV} / \mathrm{cm})$ was extracted. The band-tail filling effect was shown to be dominant in the excitation-power induced blueshifting of the PL band peak position with some possible contribution from the well-bottom flattening effect in the thickest $(4 \mathrm{~nm})$ wells. Dynamics of the band-tail filling effect was found to be modified when the incident photon energy was elevated above the barrier band gap energy, indicating an occurrence of nonthermalized distribution of the localized carriers and/or involvement of the interface states. Our results are consistent with the model of light emission from In-rich regions, which are formed at the well-barrier interface and partially extend into the barrier layer. Increased localization energy in thicker wells implies that the fluctuations of the band potential profile are enhanced with growth time.

\section{ACKNOWLEDGMENTS}

This work was partially supported by the joint Lithuanian-Latvian-Taiwan grant. A.Ž. and G.T. acknowledge the Lithuanian Ministry of Education and Science for their Fellowships.

\footnotetext{
${ }^{1}$ S. Nakamura, T. Mukai, and M. Senoh, Appl. Phys. Lett. 64, 1687 (1994). ${ }^{2}$ S. Nakamura, M. Senoh, S. Nagahama, N. Iwasa, T. Yamada, T. Matsushita, H. Kiyoku, and Y. Sugimoto, Jpn. J. Appl. Phys., Part 2 35, L74 (1996).

${ }^{3}$ S. Nakamura and G. Fasol, The Blue Laser Diode (Springer, Berlin, 1997).

${ }^{4}$ S. Chichibu, T. Azuhata, T. Sota, and S. Nakamura, Appl. Phys. Lett. 69, 4188 (1996).

${ }^{5}$ Y. Narukawa, Y. Kawakami, M. Funato, S. Fujita, S. Fujita, and S. Nakamura, Appl. Phys. Lett. 70, 981 (1997).

${ }^{6}$ S. Chichibu, T. Azuhata, T. Sota, and S. Nakamura, Appl. Phys. Lett. 70, 2822 (1997).

${ }^{7}$ P. G. Eliseev, P. Perlin, J. Lee, and M. Osiński, Appl. Phys. Lett. 71, 569 (1997).

${ }^{8}$ T. Takeuchi, S. Sota, M. Katsuragawa, M. Komori, H. Takeuchi, H. Amano, and I. Akasaki, Jpn. J. Appl. Phys., Part 2 36, L382 (1997).
}

${ }^{9}$ M. S. Minsky, S. B. Fleischer, A. C. Abare, J. E. Bowers, E. L. Hu, S. Keller, and S. P. DenBaars, Appl. Phys. Lett. 72, 1066 (1998).

${ }^{10}$ S. F. Chichibu et al., Appl. Phys. Lett. 73, 2006 (1998).

${ }^{11}$ S. Chichibu, T. Sota, K. Wada, and S. Nakamura, J. Vac. Sci. Technol. B 16, 2204 (1998).

${ }^{12}$ T. Takeuchi et al., Appl. Phys. Lett. 73, 1691 (1998).

${ }^{13}$ K. P. O'Donnell, R. W. Martin and P. G. Middleton, Phys. Rev. Lett. 82, 237 (1999).

${ }^{14}$ L.-H. Peng, C.-W. Chuang, and L.-H. Lou, Appl. Phys. Lett. 74, 795 (1999).

${ }^{15}$ T. Wang, D. Nakagawa, M. Lachab, T. Sugahara, and S. Sakai, Appl. Phys. Lett. 74, 3128 (1999).

${ }^{16}$ C. Wetzel, T. Takeuchi, H. Amano, and I. Akasaki, Phys. Rev. B 61, 2159 (2000).

${ }^{17}$ T. Wang, J. Bai, S. Sakai, and J. K. Ho, Appl. Phys. Lett. 78, 2617 (2001).

${ }^{18}$ E. Kuokstis, J. W. Yang, G. Simin, M. Asif Khan, R. Gaska, and M. S. Shur, Appl. Phys. Lett. 80, 977 (2002).

${ }^{19}$ T. Kuroda and A. Tackeuchi, J. Appl. Phys. 92, 3071 (2002).

${ }^{20}$ Y.-S. Lin et al., Appl. Phys. Lett. 77, 2988 (2000).

${ }^{21}$ D. A. B. Miller, D. C. Chemla, T. C. Damen, A. C. Grossard, W. Wiegmann, T. H. Wood, and C. A. Burrus, Phys. Rev. Lett. 53, 2173 (1984).

${ }^{22}$ A. Bykhovski, B. Gelmont, and M. Shur, J. Appl. Phys. 74, 6734 (1993).

${ }^{23}$ J. A. Davidson, P. Dawson, T. Wang, T. Sugahara, J. W. Orton, and S. Sakai, Semicond. Sci. Technol. 15, 497 (2000).

${ }^{24}$ R. Singh, D. Doppalapudi, T. D. Moustakas, and L. T. Romano, Appl. Phys. Lett. 70, 1089 (1997).

${ }^{25}$ N. A. El-Masry, E. L. Piner, S. X. Liu, and S. M. Bedair, Appl. Phys. Lett. 72, 40 (1998).

${ }^{26}$ K. Kazlauskas et al., Appl. Phys. Lett. 82, 4501 (2003).

${ }^{27}$ K. Kazlauskas et al., Appl. Phys. Lett. 83, 3722 (2003).

${ }^{28}$ F. Bernardini, V. Fiorentini, and D. Vanderbilt, Phys. Rev. B 56, R10024 (1997).

${ }^{29}$ P. Lefebvre et al., Appl. Phys. Lett. 78, 1252 (2001).

${ }^{30}$ A. Hangleiter, F. Hitzel, S. Lahmann, and U. Rossow, Appl. Phys. Lett. 83, 1169 (2003).

${ }^{31}$ F. Della Sala, A. Di Carlo, P. Lugli, F. Bernardini, V. Fiorentini, R. Scholz, and J.-M. Jancu, Appl. Phys. Lett. 74, 2002 (1999).

${ }^{32}$ Y.-Y. Chung et al., J. Appl. Phys. 93, 9693 (2003).

${ }^{33}$ M. Hao, H. Ishikawa, T. Egawa, C. L. Shao, and T. Jimbo, Appl. Phys. Lett. 82, 4702 (2003).

${ }^{34}$ H. Schömig, S. Halm, A. Forchel, G. Bacher, J. Off, and F. Scholz, Phys. Rev. Lett. 92, 106802 (2004).

${ }^{35}$ M. E. Aumer, S. F. LeBoeuf, B. F. Moody, and S. M. Bedair, Appl. Phys. Lett. 79, 3803 (2001).

${ }^{36}$ A. Bell, J. Christen, F. Bertram, F. A. Ponce, H. Marui, and S. Tanaka, Appl. Phys. Lett. 84, 58 (2004).

${ }^{37}$ D. P. Wang, C. T. Chen, H. Kuan, S. C. Shei, and Y. K. Su, J. Appl. Phys. 78, 2117 (1995)

${ }^{38}$ C. Wetzel, T. Takeuchi, S. Yamaguchi, H. Katoh, H. Amano, and I. Akasaki, Appl. Phys. Lett. 73, 1994 (1998).

${ }^{39}$ W. Shan et al., J. Appl. Phys. 84, 4452 (1998).

${ }^{40}$ A. Dmitriev and A. Oruzheinikov, J. Appl. Phys. 86, 3241 (1999).

${ }^{41}$ P. C. Chow, Am. J. Phys. 40, 730 (1972).

${ }^{42}$ C. G. Van der Walle and J. Neugebauer, Appl. Phys. Lett. 70, 2577 (1997).

${ }^{43}$ Y. C. Yeo, T. C. Chong, and M. F. Li, J. Appl. Phys. 83, 1429 (1998).

${ }^{44}$ M. Suzuki, T. Uenoyama, and A. Yanase, Phys. Rev. B 52, 8132 (1995).

${ }^{45}$ K. S. Lee, D. H. Yoon, S. B. Bae, M. R. Park, and G. H. Kim, ETRI J.1225-6463 24, 270 (2002).

${ }^{46}$ A. M. Cruz Serra and H. Abreu Santos, J. Appl. Phys. 70, 2734 (1991).

${ }^{47}$ A. Hangleiter, J. Lumin. 87-89, 130 (2000).

${ }^{48}$ S. Miasojedovas et al., Phys. Status Solidi C 0, 2610 (2003).

${ }^{49}$ A. Satake, Y. Masumoto, T. Miyajima, T. Asatsuma, F. Nakamura, and M. Ikeda, Phys. Rev. B 57, R2041 (1998).

${ }^{50}$ S. Juršènas, G. Kurilčik, and A. Žukauskas, Phys. Rev. B 58, 12937 (1998).

${ }^{51}$ L. J. Brillson, T. M. Levin, G. H. Jessen, and F. A. Ponce, Appl. Phys. Lett. 75, 3835 (1999). 